\title{
Systemic Steroid-Pulse Therapy in a Patient with Idiopathic Choroidal Detachment: A Case Report
}

\author{
Takashi Kanamoto Michiya Takamatsu \\ Department of Ophthalmology and Visual Sciences, Graduate School of \\ Biomedical Sciences, Hiroshima University, Hiroshima, Japan
}

\section{Key Words}

Cataract surgery · Idiopathic choroidal detachment · Systemic steroid-pulse therapy

\begin{abstract}
Aim: To report on the effectiveness of systemic steroid-pulse therapy in treating idiopathic choroidal detachment.

Case Presentation: Our patient developed idiopathic choroidal detachment after cataract surgery for pseudoexfoliation glaucoma and glaucoma filtration surgery. Systemic steroidpulse therapy was performed, and the choroidal detachment resolved completely.

Conclusion: We suggest systemic steroid-pulse therapy as a treatment option for idiopathic choroidal detachment with low intraocular pressure.
\end{abstract}

\section{Introduction}

Choroidal detachment can be found in several ocular diseases, e.g. uveitis, hypotony, uveal effusion, and rhegmatogenous retinal detachment [1]. However, the diagnosis of the cause for choroidal detachment from clinical symptoms, signs, and routine investigations can sometimes be difficult.

Choroidal detachments are commonly treated with steroid therapy, scleral needling, scleral incision, or antibody therapy [2]. The recovery rate of choroidal detachment with a rhegmatogenous detachment is increased by the use of oral steroids before primary vitrectomy [3]. A retrobulbar injection of triamcinolone acetonide has been shown to be effective in cases of polypoidal choroidal vasculopathy and Crohn's disease-induced choroiditis with choroidal detachment $[4,5]$. The combined retinal and choroidal detachments in an eye with Vogt-Koyanagi-Harada disease was resolved after systemic corticosteroid therapy [6]. In another case, an annular choroidal detachment 
with optic disc swelling in an eye with Vogt-Koyanagi-Harada disease was also resolved by systemic steroid therapy [7]. These case reports demonstrated that systemic steroid therapy is effective for choroidal detachments.

\section{Case Presentation}

An 81-year-old man complained of disturbances in the visual field of his left eye. He did not have any systemic illness, but his blood pressure was approximately $140 / 75 \mathrm{~mm} \mathrm{Hg}$ with amlodipine besylate and aspirin. There was no family history of any systemic or ocular diseases. Ten years earlier, he had been diagnosed with pseudoexfoliation glaucoma in his left eye. He had undergone lasertrabeculoplasty and been treated postoperatively with topical $0.05 \%$ latanoprost, $0.5 \%$ timolol, and $1.0 \%$ dorzolamide hydrocholide in his left eye. The intraocular pressure (IOP) in the left eye fluctuated between 14 and $25 \mathrm{~mm} \mathrm{Hg}$.

Five years later, trabeculectomy was performed on his left eye to decrease the IOP because of a worsening of his visual fields, and bleb-revision surgery was performed after that. The postoperative IOP in the left eye was controlled in the low teens, and cataract surgery was performed under subTenon's anesthesia by injection of $0.5 \mathrm{ml} 2.0 \%$ lidocaine using a blunt needle on the left eye because of a decrease in his visual acuity. Twenty days after the cataract surgery, he returned complaining of pain in his left eye.

The IOP in his left eye was $8 \mathrm{~mm} \mathrm{Hg}$ as measured with a Goldmann applanation tonometer. The filtrating bleb was encapsulated and the anterior chamber depth was normal. Slit-lamp biomicroscopy showed no inflammation in the anterior segment of the left eye and the intraocular lens was correctly positioned. Gonioscopy showed that the anterior chamber angle was wide with no anterior synechia.

Ocular fundus examination showed a focal choroidal detachment in the temporal area of the left eye without a retinal detachment. One month later, the IOP in the left eye was still $8 \mathrm{~mm} \mathrm{Hg}$ and the choroidal detachment had extended to the upper-nasal quadrant (fig. 1a). B-mode echography showed that the choroidal detachment was not associated with a choroidal hemorrhage, and ophthalmoscopy showed no choroidal bleeding, vitreoretinal inflammation, or exudative lesions. In addition, ultrasound biomicroscopy showed no ciliary body laceration or ciliary body detachment. We concluded that the choroidal detachment was idiopathic.

To try to reduce the subchoroidal fluid, $20 \mathrm{mg} /$ day of prednisolone was used for 20 days, but the area of choroidal detachment worsened and progressed to the temporal area (fig. 1b, c). Because it was suspected that the dose of steroids had been too low to have an effect on choroidal detachment, steroid-pulse therapy (methylprednisolone sodium succinate: 1,000 mg/day) was administered for 3 days, finally. After the steroid-pulse therapy, the IOP in the left eye was increased to $11 \mathrm{~mm} \mathrm{Hg}$ and the choroidal detachment gradually decreased (fig. 2 a). The amount of steroids was gradually decreased. After 1 month the choroidal detachment was hardly visible, except for the peripheral margin of temporal area, and the IOP in the left eye was $15 \mathrm{~mm} \mathrm{Hg}$. The choroidal detachment had completely resolved 2 months after the steroid-pulse therapy was begun, and the IOP in the left eye was $20 \mathrm{~mm} \mathrm{Hg}$ at the same time (fig. $2 \mathrm{~b}, \mathrm{c}$ ).

Written informed consent was obtained from the patient for publication.

\section{Discussion}

Our case showed no signs of scleritis, and there was neither inflammation nor exudative lesion in the ocular fundus. A retinal detachment was not observed, nor was a movement of the subchoroidal fluid seen during the fundus examination. These findings indicated that uveal effusion can be excluded as the cause for the choroidal detachment. Thus, we concluded that the patient had idiopathic choroidal detachment. 
Although it was suspected that our case might have uveitis-induced choroidal detachment, the appearance and shape of the detachment was not typical of those reported for uveitis. We did not know whether choroidal detachment had been due to hypotony or not, because large choroidal detachments are usually observed in cases with IOPs less than $5 \mathrm{~mm} \mathrm{Hg}$, shallow depth of anterior chamber, and leakage of aqueous humor, and these alterations were not found in our case. We chose systemic steroid therapy initially to decrease the volume of the subchoroidal fluid without treating the choroidal detachment and found that the choroidal detachment completely disappeared. The IOP increased to $15 \mathrm{~mm} \mathrm{Hg} 1$ month after the steroid-pulse therapy, and it seemed that the choroidal detachment was dependent on the IOP. This might suggest that the steroid-pulse therapy was effective because the choroidal detachment was accompanied by low IOP.

One limitation of this case report is that there is no evidence that the treatment had a causal effect on the outcome and that the condition would not have resolved spontaneously. Therefore, we cannot conclude that systemic steroid therapy is effective, recommending it for other similar cases. But, this case suggests that systemic steroid therapy be considered as a treatment option for eyes with choroidal detachment with low IOP.

\section{Disclosure Statement}

The authors declare that they have no competing interests. 

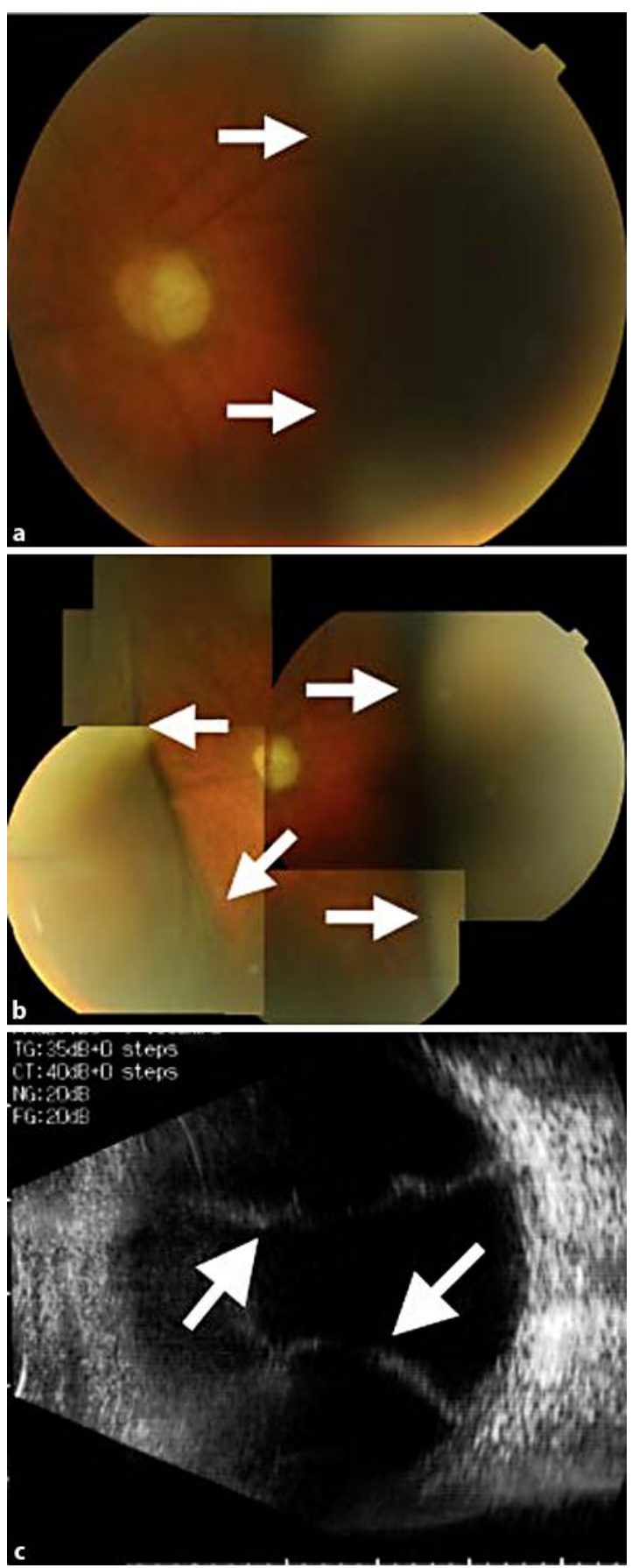

Fig. 1. Fundus photograph and ultrasonographic image of choroidal detachment. a Fundus photograph showing the choroidal detachment (arrows) in the temporal area of the left eye. $\mathbf{b}$ A progression of the choroidal detachment (arrows) can be seen. c B-mode echographic image showing choroidal detachment without choroidal hemorrhage. 


(2)
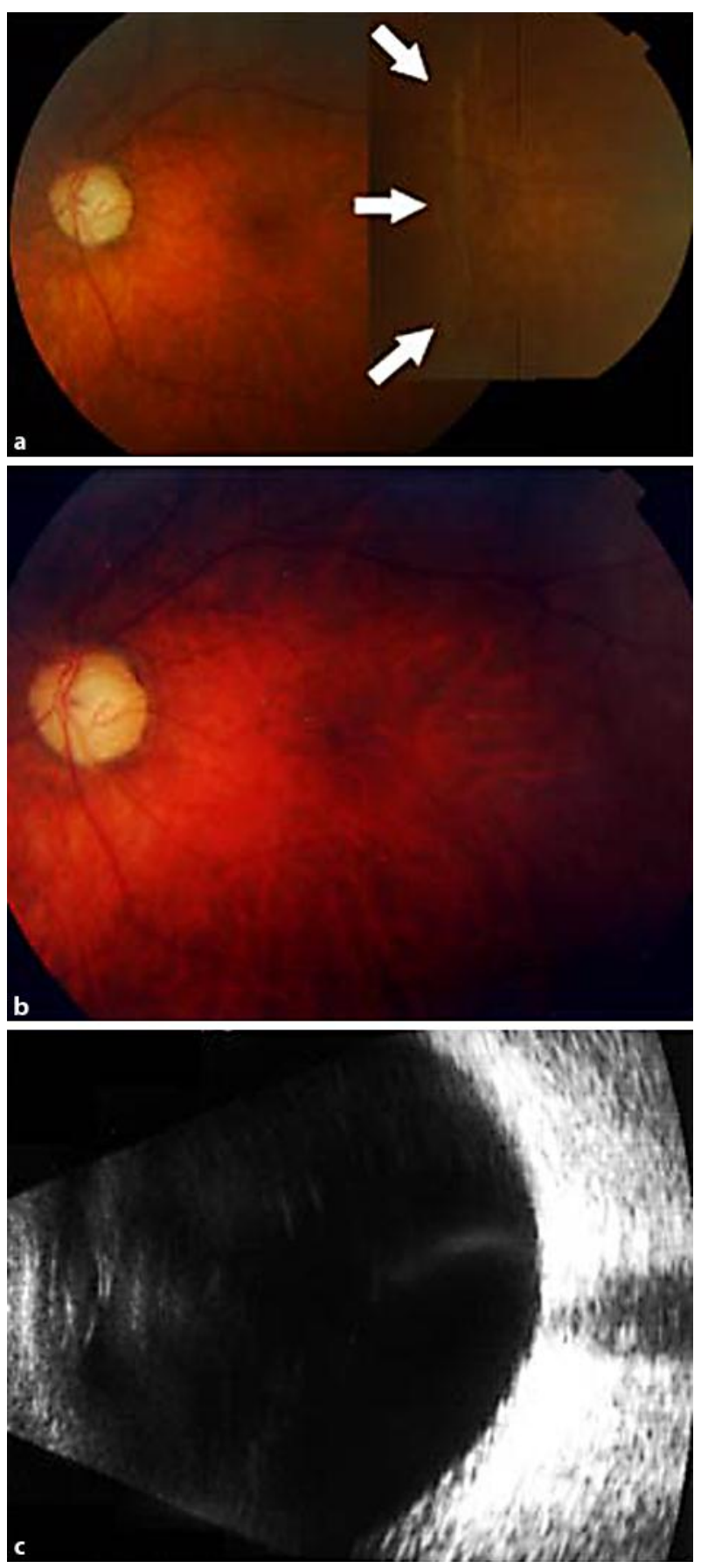

Fig. 2. Disappearance of the choroidal detachment. a Fundus photograph showing that the choroidal detachment cannot be seen and only the demarcation line is visible (arrows). $\mathbf{b}, \mathbf{c}$ Choroidal detachment cannot be seen. 


\section{References}

1 Slagle WS, Boothe KH, Musick AN, Eckermann DR, Slagle AM: Idiopathic orbital inflammatory syndrome without pain: a case report. Optometry 2010;81:146-152.

2 Matthews BN, Stavrou P: Bilateral combined retinal and choroidal detachment in antineutrophil cytoplasmic antibody-positive scleritis. Acta Ophthalmol Scand 2003;81:405-407.

-3 Sharma T, Gopal L, Kasinathan N, Shah NA, Sulochana KN, Miriam KC, Arvind K, Ramakrishnan S, Sukumar B: Primary vitrectomy for combined rhegmatogenous retinal detachment and choroidal detachment with or without oral corticosteroids: a pilot study. Retina 2005;25:152-157.

4 Okubo A, Ito M, Kamisasanuki T, Sakamoto T: Visual improvement following trans-Tenon's retrobulbar triamcinolone acetonide infusion for polypoidal choroidal vasculopathy. Graefes Arch Clin Exp Ophthalmol 2005;243:837-839.

-5 Vianna RN, Ozdal PC, Deschénes J: Multifocal choroiditis - an unusual finding in Crohn's disease. Eur J Ophthalmol 2004;14:345-349.

-6 Watanabe K, Kato T, Hayasaka S: Concurrent bilateral posterior scleritis and Vogt-Koyanagi-Harada disease in a patient with positive rheumatoid factor. Ophthalmologica 1996;211:316-319.

-7 Hirooka K, Shiraga F, Tanaka S, Baba T, Mandai H: Risk factors for elevated intraocular pressure after trans-tenon retrobullbar injections of triamcinolone. Jpn J Ophthalmol 2006;50:235-238. 